\title{
In search of phonotactic preferences
}

\author{
Paula Orzechowska \\ Faculty of English, Adam Mickiewicz University, Poznań \\ paulao@wa.amu.edu.pl
}

\begin{abstract}
The objective of this contribution is to provide an analysis of consonant clusters based on the assumption that phonotactic preferences are encoded in phonological features of individual segments forming a cluster. This encoding is expressed by a set of parameters established for the following features: complexity, place of articulation, manner of articulation and voicing. On the basis of empirically observed tendencies of feature distribution and co-occurrence, novel phonotactic preferences for English word-initial consonant clusters are proposed. Statistical methods allow us to weigh the preferences and determine a ranking of phonological features in cluster formation.
\end{abstract}

Keywords: phonotactic preferences; feature weighing and ranking; cluster analysis; PCA.

\section{Phonotactic models}

Phonotactics is concerned with conditions under which segments combine to form larger units, such as syllables or words. The most basic sequencing of sounds found across languages is that of alternating consonants $(\mathrm{C})$ and vowels (V), while combinations of adjacent consonants are considered rare, dispreferred and subject to severe phonotactic restrictions (Greenberg 1978; Maddieson 2011). In order to account for the occurrence and abundance of consonant clusters in some linguistic systems, phonological theory has relied on the generalizing principle of sonority, defined in terms of the degree of opening of the vocal tract and the ensuing relative loudness of segments (Goldsmith 1990; Ladefoged 2011). Despite there being numerous sonority hierarchies proposed over the past century, all posit increasing sonority from obstruents through sonorants to vowels, and all are based on the manner of articulation features. The manner categories vary in terms of phonological 
detail, ranging from a broad class of obstruents (Clements 1990; Zec 1995) to specific manner distinctions between, say, liquids $/ \mathrm{r} /$ and $/ 1 /$ (Jespersen 1904; Wiese 1988; Parker 2002). Some hierarchies go beyond the manner features. Jespersen (1904), Vennemann (1988) and Parker (2002) not only distinguish between voiced and voiceless obstruents, but also vowels of different height (high, mid, low).

The structure of cluster constituents is universally specified by a welldefined sonority profile. The Sonority Sequencing Generalisation (SSG, Selkirk 1984), traditionally applied to a syllable, requires that adjacent consonants increase in sonority from the syllable margins towards the nucleus. This principle evaluates consonant clusters depending on their phonological make-up and position they occupy in the syllable. For example, English onset /p1/ and / $\mathrm{r} /$ (plan, shrink) as well as coda /lp/ and /rj/ (help, harsh) have the predicted decreasing sonority profile from the vocalic peak outward, while their mirror images, i.e. onset $/ \mathrm{lp} /$ and coda $/ \mathrm{pl} /$ violate the SSG. This principle explains some phonotactic constraints, whereby the ill-formed combinations /lp-/, /rf-/, /-pl/, /- $\mathrm{r} /$ are simply not attested in English. However, sonority and the SSG have notoriously been proven unable to account for existent clusters such as $s+$ stop (+liquid) found in e.g. skin, speak, split, string. The structure of CCCs is captured in theoretical non-linear approaches to the syllable (cf. Goad 2011, 2012; Kaye 1992; Kaye et al. 1990; Steriade 1982).

A phonotactic model which makes much finer predictions in measuring degrees of cluster preferability is Net Auditory Distance (NAD, DziubalskaKołaczyk 2009, 2014). NAD encompasses several phonological features beyond sonority, namely manner of articulation (MOA), place of articulation $[\mathrm{POA}]$, and voicing (or sonorant/obstruent distinction) (S/O). Calculations are performed on the basis of well-formedness conditions for CCs and CCCs depending on a word position in the following way:

$$
\mathrm{NAD}=|\mathrm{MOA}|+|\mathrm{POA}|+|\mathrm{S} / \mathrm{O}|
$$

where $|\mathrm{MOA}|,|\mathrm{POA}|$ and $|\mathrm{S} / \mathrm{O}|$ stand for absolute values of difference in distance between neighbouring sounds. The conditions account not only for distances between pairs of consonants (e.g. $\mathrm{C} 1 \mathrm{C} 2$ for a double cluster, $\mathrm{C} 1 \mathrm{C} 2$ and $\mathrm{C} 2 \mathrm{C} 3$ for a triple cluster), but also for distances between a consonant and a neighbouring vowel (e.g. $\mathrm{C} 2 \mathrm{~V}, \mathrm{C} 3 \mathrm{~V}$ ). To compare with the previouslymentioned examples, NAD evaluates initial /st/ and /sp/ clusters as dispre- 
ferred, while $/ \mathrm{skw} /$ and $/ \mathrm{str} /$ are preferred. This difference is motivated by perceptual criteria, according to which, in order for a cluster to be preserved, the distance between a pair of consonants should be larger than between a consonant and a vowel. In this way, the NAD principle offers a more detailed analysis of consonant sequencing than sonority does.

\subsection{An alternative approach}

Phonotactic models generally provide a top-down analysis of clusters; they rely on deductive premises on the general syllable or word structure, and specify conditions under which a sequence of consonants can be considered good or preferred. Moreover, since the existing approaches aim at evaluating cluster structure, rather than looking into it, they make use of universal wellformedness predictions which can be easily applied to all phonotactic grammars, and in which all phonological features contribute equally to the final result (i.e. a cluster being well- or ill-formed). The goal of this analysis is to complement the existing research with specific phonotactic preferences in English. These preferences are found on the basis of an in-depth quantitative and statistical analysis of phonological features of segments forming clusters. This approach is inductive; it uses no superimposed formalized constraints that tend to overshadow the role of place, manner and voice features in cluster formation. Therefore, it allows us to trace phonotactic preferences, and establish weight of phonological features. This paper draws on the original idea of Orzechowska and Wiese (2011) who reconstructed the sonority hierarchy into nine phonotactic preferences for word-initial clusters in German. In terms of methodology, the present contribution provides a refinement of the overall procedure advocated in Orzechowska and Wiese (2015), an extension of their earlier work, and tested on Polish and German word-initial phonotactics.

Orzechowska and Wiese (2015) conducted a meticulous analysis of Polish and German word-initial clusters in terms of 15 parameters referring to four broad dimensions; complexity, place of articulation, manner of articulation and voicing, with a view to establishing rankings of clusters. Ranks are arrived at by summing up individual scores that each cluster obtains for parameters (1-15). The present analysis differs from Orzechowska and Wiese (2015) in several ways. Firstly, I suggest providing a more detailed analysis of phonotactics by increasing the number of parameters used in cluster de- 
scription to 19 (cf. Section 3.3). Secondly, instead of arriving at a ranking of clusters, I posit a ranking of phonological features contributing to cluster formation (cf. Section 4). Methods of statistical testing applied to the data make it possible to establish weights of the parameters and phonological features that the parameters represent (cf. Section 4.1). These weights correspond to the role that specific parameters and features play in consonant clustering in English.

\section{Featuretactics}

The present paper is based on the assumption that phonotactic preferences are encoded in phonological features of segments within a cluster and that phonotactic constraints can be formulated on the basis of an in-depth analysis of these features. Therefore, I propose the term feature-tactics, in opposition to phono-tactics, as a branch of phonology studying the relation between phonological features (rather than between phonemes in phono-tactics) within and across units, here within and across segments forming clusters. Featuretactics thus specifies the distribution and co-occurrence of distinctive features (in particular, parameters representing these features) in clusters, and the role (here expressed by rankings and weight) that they play in cluster formation. The focal point of the reasoning adopted here is that a specific feature setting expressed by a set of preferences is responsible for the structure of consonant clusters in a given language and word position. The same set of phonological features (complexity, place, manner, voice) is universally available to all languages; however, each language selects a feature or a subset of features in the construction of clusters, which decide on the idiosyncratic character of clusters in a given phonological system.

This analysis not only expands on the existing approaches to phonotactic complexity (Hoole et al. 2012), but also complements them. Featuretactics is not based on any a priori assumptions on cluster length, cluster structure or word position in which a cluster occurs. It does not assume a prime role of linguistic universals as principles imposing structural restrictions. Neither does it state predictions under which a cluster should be considered wellformed or preferred. The inductive quantitative method adopted here allows to make observations on the basis of a bottom-up and detailed analysis of the arrangement and distribution of features and parameters representing these features in clusters. Featuretactics has thus the potential of revealing what 
consonant clusters in a given system are like, rather than what they should be like, leading to novel insights about language-specific and typologicallybased phonotactic preferences.

The starting point for proposing featuretactics is the study of Orzechowska and Wiese (2015). The side result of their analysis is that Polish and German use a different set of parameters (distinctive features) in the formation of word-initial clusters. The authors suggest that Polish phonotactics prefers the place of articulation and voicing features (i.e. presence of one or more coronal(s), a coronal cluster-finally, voice agreement across segments in a cluster), while German forms clusters based on several features which conspire to achieve a sonority-based profile (i.e. size, increase in articulatory opening, voicing cluster-initially). This observation is taken as a leading thought for the new line of research proposed below. Since parameters are believed to have the status of typologically-relevant preferences, to supplement the study conducted for Polish and German, this paper tests the method on English word-initial clusters.

The hypothesis put forward here is that the established parameters and phonological features (complexity, place, manner, voice) which these parameters represent differ in weight cross-linguistically. As a result, each language operates on its own unique feature setting which is an expression of the parameters' weight. This feature setting determines the phonological composition of clusters depending on a position within a word (initial, final). In this sense, the reasoning adopted here corresponds with feature-based approaches which look into the internal structure of segments (Clements 1985; Goldsmith 1990; Harris 1990), while weighing features goes in line with Optimality Theory rankings (Dresher 2009; Morelli 1999; Rochon 2000). The present study is restricted to the word-initial context, which is motivated by previous contributions stipulating position-dependent constraints (Hyman 1977; Hayes 1989).

\section{The study}

\subsection{English cluster inventory}

To obtain a comprehensive inventory of word-initial clusters, several works on English phonotactics (Gimson 2014; Roach 2006; Trnka 1966) were compared. The accounts report on 52-65 CCs and CCCs, including sequences 
found in rare words and recent imports. The target list used in this study contains clusters which appeared in all or most sources, and excludes rare forms (/bd smj vr/ in bdellium, smew, vroom), loans (/klw kv $\int \mathrm{m} \int \mathrm{n} \int \mathrm{p} \int \mathrm{w} \mathrm{vw} /$ in cloisonne, kvass, schmalz, schnapps, Spiel, Schwepps, voyeur), and proper nouns (/kn pn pt sr tl vl/ in Knossos, Pnom Penh, Ptah, Sri Lanka, Tlingit, Vladimir). Although some of these clusters are found in phonetic dictionaries (Wells 2009; Jones 2011), they tend to be simplified with an anaptyxic or a prothetic vowel. The inclusion of such clusters into the dataset, similarly to clusters of a notably foreign status, would blur the phonological picture of left edge phonotactics in English. Additionally, this paper is focused on a structural description of clusters, rather than, e.g. their stylistically-driven modification. The final list of 50 clusters $(41 \mathrm{CCs}, 9 \mathrm{CCCs})$ serves as an input for testing the model.

Table 1. Word-initial clusters in English.

\begin{tabular}{|c|c|}
\hline $\mathrm{CC}$ & $\mathrm{CCC}$ \\
\hline 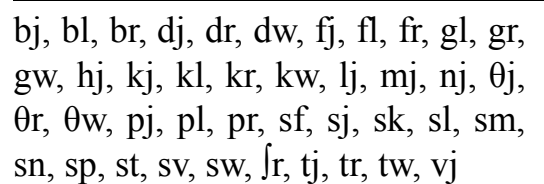 & $\begin{array}{l}\text { skj, skl, skr, skw, spj, spl, spr, stj, } \\
\text { str }\end{array}$ \\
\hline
\end{tabular}

\subsection{Methodology}

The present contribution provides an extension of the overall procedure established by Orzechowska and Wiese (2015), in which dimensions are proposed. Since dimensions correspond with phonological features of consonant classification (e.g. voice), the latter term will be used throughout the paper to avoid confusion. Each phonological feature is instantiated by parameters (e.g. voice agreement; voicing in initial C) and patterns referring to a specific realization of a feature - its presence (e.g. total/partial/no agreement) or its distribution (e.g. [ \pm voice] cluster-initially). Patterns can have the form of descriptive labels and numerical values. The number of clusters adhering to a particular pattern (expressed by percentages) is transformed into percentage scores (from 0 to 1) calculated over all clusters. The sum of the 19 scores reveals the extent to which a given cluster follows all or a set of parameters. All clusters 
display a particular pattern for each parameter, reflected by a percentage score. Some patterns of phonological organization can have the status of a preference (e.g. [+voice] cluster-finally) depending on the degree to which all clusters adhere to a particular pattern. For instance, if the proportion of [ - voice] and [+voice] segments cluster-finally is $40: 10$ (total $=50$ clusters), clusters ending with a voiceless $\mathrm{C}$ score $0.8(=80 \%)$, while clusters ending with a voiced $\mathrm{C}$ are assigned a score of $0.2(=20 \%)$ for the voicing in final $C$ parameter. If all clusters with no exception follow a particular parameter, each cluster receives a score equal to $1(=100 \%)$. Such an analysis requires a rich preferably exhaustive - cluster inventory. A pattern which can be seen as a tendency, i.e. holding over $60 \%$ of clusters, is considered to have the status of a phonotactic preference (for more, cf. 3.3.1). The established preferences provide insights into a specific feature setting and are derived from quantitative empirical evidence by counting clusters adhering to a given pattern.

\subsection{Proposing parameters}

The original study by Orzechowska and Wiese (2015) uses 15 parameters. In order to ensure a more exhaustive and detailed analysis, the cluster description is here captured by 19 categories (cf. Table 2 below). The new parameters involve: directionality (the direction of the tongue movement from the first to the last consonant, 5), glottal $C$ (the number of glottal consonants in a cluster, 9), initial $C$ (manner of articulation cluster-initially, 15), and final $C$ (manner of articulation cluster-finally, 16). The disproportion in the number of parameters is attributed to the fact that some of them can be expressed by a binary distinction while others by employing a larger set of categories.

Table 2. The 19 parameters of cluster description.

\begin{tabular}{llll}
\hline Complexity & Place & Manner & Voice \\
\hline 1. Size & 4. Distance & 12. Distance & 17. Initial C \\
2. Compositionality & 5. Directionality & 13. Opening & 18. Final C \\
3. Identity avoidance & 6. Labial C & 14. Obstruents & 19. Agreement \\
& 7. Coronal C & 15. Initial C & \\
& 8. Dorsal C & 16. Final C & \\
& 9. Glottal C & & \\
& 10. Initial C & & \\
11. Final C & & \\
\hline
\end{tabular}


Among eight place parameters, four express a distinction into broad articulatory types, i.e. labial, coronal, dorsal and glottal C. An equivalent in terms of the manner feature is represented by just a single parameter called obstruents, which captures a two-fold division into obstruents and sonorants. As a result, the same aspect of phonotactic grammar is tested by means of one manner parameter (14) and four place parameters (6-9). This discrepancy is unavoidable given the goal of examining the same context (if possible) for all the features. In contrast, some parameters are directly comparable across features. For example, the parameters of distance proposed for the place and manner classes have their closest voice equivalent in voice agreement. In other words, the distance in voicing is present when adjacent segments are neither uniformly voiced nor voiceless. The subsequent sections of the paper focus on the description of each parameter.

\subsubsection{Complexity}

For the feature of complexity, three parameters are proposed. Cluster size (1) specifies the number of segments within a cluster (i.e. CC, CCC). Cluster compositionality (2) refers to the property of complex clusters being decomposable into simpler constituents (e.g. a CCC can be formed of two existing CCs). These parameters correspond with Greenberg's [1978] universals on cluster size and resorvability, respectively. As a modification of the Obligatory Contour Principle (Leben 1973; McCarthy 1986), parameter (3) referring to identity avoidance tests the occurrence of identical (not necessarily adjacent) segments within a cluster. "Identity" is here understood in terms of an identical place, manner and voice profile of at least two segments in a cluster (this is the case, e.g. in Polish initial clusters /fsf sks/). The results of the analysis are given in Table 3, where shaded areas mark a pattern which is interpreted as a phonotactic preference. A pattern represented by more than one half of clusters (here, the threshold of $60 \%$ ) is considered to have the status of a preference. The more clusters follow a particular pattern, the higher the percentage score, and the stronger the preference. Scores higher than $0.8(\geq$ $80 \%)$ and equal to $1(=100 \%)$ are seen as a strong and absolute preference respectively. For this and the remaining features, each table reports on the number of clusters adhering to a particular pattern (labelled No) and percentages calculated for the total of 50 word-initial clusters (labelled \%). 
Table 3. The complexity feature.

\begin{tabular}{lllrr}
\hline & Parameters & Patterns & No & $\%$ \\
\hline 1. & Size & CC & 41 & 82 \\
& & CCC & 9 & 18 \\
2. & Compositionality & full & 50 & 100 \\
3. & Identity avoidance & total & 50 & 100 \\
\hline
\end{tabular}

As is shown, English initial phonotactics prefers shorter (CC) and fully compositional clusters. All three-consonant clusters /skj skl skr skw spj spl spr stj str/ are composed of existing $\mathrm{C} 1 \mathrm{C} 2 / \mathrm{sk}$ sp st/ and $\mathrm{C} 2 \mathrm{C} 3 / \mathrm{kj} \mathrm{kl} \mathrm{kr} \mathrm{kw} \mathrm{pj} \mathrm{pl} \mathrm{pr} \mathrm{tj}$ tr/, while all CCs contain single segments belonging to the English consonant inventory. Finally, English contains no initial cluster formed of two or more identical segments. The results lead to the formulation of a strong preference (here expressed by $82 \%$ ) for parameter (1), and absolute preferences for (2) and (3).

\subsubsection{Place of articulation}

The feature of place of articulation is represented by eight parameters. The general description of consonants in terms of place is based on Gimson (2014) and Roach (2004). Parameter (4) is derived from NAD (DziubalskaKołaczyk 2009, 2014), a model in which the concept of distances is employed systematically. Here, a distance of one is assigned to all adjoining place features, and holds between the bilabial - labio-dental - inter-dental alveolar - post-alveolar - palatal - velar - glottal articulations. For instance, the smallest distance equal to zero is found in clusters with identical place features such as alveolar + alveolar in /st sn sl/, while the largest distance of five is exemplified by bilabial + palatal articulation in /pj bj mj/. For labiovelar $/ \mathrm{w} /$, the mean is counted for the labial and velar articulations separately. Clusters $/ \mathrm{kw} \mathrm{gw} /$ are assigned distance $=3$, resulting from the computation of the respective distances: $($ velar $/ \mathrm{k} /$-velar $/ \mathrm{w} /)+($ velar $/ \mathrm{k} /$-bilabial $/ \mathrm{w} /)=$ $(0+6) / 2=3$. A similar averaging procedure is applied to CCCs. For instance, /spr/ has the following distances: C1-C2 (alveolar-bilabial) and C2-C3 (bilabial-postalveolar) $=(3+4) / 2=3.5$. Decimal numbers are rounded to the closest whole number, yielding the final distance of four. 
Apart from the specific articulations given above, the place feature also uses broader categories. The classification of segments into labial (bilabial, labio-dental), coronal (inter-dental, alveolar, post-alveolar), dorsal (palatal, velar) and glottal is based on Ladefoged (2011). These distinctions are used in parameters (6-9) that determine the number of segments of a particular type in a cluster, as well as parameters (10-11) that specify a type of a segment found cluster-initially (C1) and cluster-finally ( $\mathrm{C} 2$ in $\mathrm{C} 1 \mathrm{C} 2, \mathrm{C} 3$ in $\mathrm{C} 1 \mathrm{C} 2 \mathrm{C} 3$ ). Moreover, in order to account for double articulation of $/ \mathrm{w} /$, an additional labio-dorsal category is posited. Since both the labial and dorsal articulations are simultaneous, $/ \mathrm{w} /$ is subsumed under a separate class to facilitate calculations.

Finally, directionality (5) specifies the tongue movement throughout a cluster. For CCs, possible patterns involve: a forward gesture towards a more front articulation (e.g. glottal $\rightarrow$ palatal /hj/, velar $\rightarrow$ alveolar /gl/, alveolar $\rightarrow$ bilabial /sp/), a backward gesture towards a more retracted articulation (e.g. bilabial $\rightarrow$ palatal $/ \mathrm{pj} /$, labio-dental $\rightarrow$ alveolar $/ \mathrm{fl} /$, alveolar $\rightarrow$ velar $/ \mathrm{sk} /$ ), and a level gesture where no tongue movement is involved (e.g. alveolar $\rightarrow$ alveolar /sl/, post-alveolar $\rightarrow$ post-alveolar /fr/). For CCCs, backward (e.g. alveolar $\rightarrow$ alveolar $\rightarrow$ palatal $/ \mathrm{stj} /$, alveolar $\rightarrow$ alveolar $\rightarrow$ post-alveolar /str/) and mixed (e.g. alveolar $\rightarrow$ bilabial $\rightarrow$ palatal $/ \mathrm{spj} /$, alveolar $\rightarrow$ velar $\rightarrow$ post-alveolar /skr/) articulations are found. The latter pattern is also used to define clusters containing /w/ (e.g. alveolar $\rightarrow$ labio-velar $/ \mathrm{dw}$ sw tw $\theta \mathrm{w} /$, alveolar $\rightarrow$ velar $\rightarrow$ labio-velar /skw/) due to double articulation of the glide, which involvs two movements in opposite directions; towards the bilabial (forward) and velar (backward) regions. The only exceptions constitute $/ \mathrm{kw} /$ and $/ \mathrm{gw} /$, which are subsumed under the forward class, resulting from the combination of two articulatory types: level (velar $\rightarrow$ velar) and forward (velar $\rightarrow$ bilabial).

Clear preferences can be formulated for glottal and coronal segments. Firstly, glottal consonants are largely dispreferred (9). In fact, there is only one cluster exemplifying this place feature, namely /hj/ in huge. Another clear preference can be formulated for coronality in the cluster-initial position (10). No preferences can be stated for labial (6) and dorsal (8) segments. Similarly, place distances (4) yield no conclusive results. Generally, initial clusters seem to prefer medial distances (e.g. 2 : $/ \mathrm{dj} \mathrm{kr} \mathrm{sv} /, 3$ : $/ \theta \mathrm{j} \mathrm{pl} \mathrm{sm/),} \mathrm{with}$ large and small distances constituting a minority. This distribution is similar to normal distribution; relatively few clusters with a distance of zero or five can be created due to the limitations of the segment inventory. To recapitulate, 
Table 4. The place of articulation feature.

\begin{tabular}{|c|c|c|c|c|}
\hline & Parameters & Patterns & No & $\%$ \\
\hline \multirow{6}{*}{4.} & \multirow{6}{*}{ Distance } & 0 & 4 & 8 \\
\hline & & 1 & 5 & 10 \\
\hline & & 2 & 13 & 26 \\
\hline & & 3 & 19 & 38 \\
\hline & & 4 & 6 & 12 \\
\hline & & 5 & 3 & 6 \\
\hline \multirow{4}{*}{5.} & \multirow{4}{*}{ Directionality } & forward & 12 & 24 \\
\hline & & backward & 23 & 46 \\
\hline & & level & 4 & 8 \\
\hline & & mixed & 11 & 22 \\
\hline \multirow{2}{*}{6.} & Number & 0 & 25 & 50 \\
\hline & of labial C & 1 & 25 & 50 \\
\hline \multirow{4}{*}{7.} & \multirow{4}{*}{$\begin{array}{l}\text { Number } \\
\text { of coronal C }\end{array}$} & 0 & 9 & 18 \\
\hline & & 1 & 28 & 56 \\
\hline & & 2 & 12 & 24 \\
\hline & & 3 & 1 & 2 \\
\hline \multirow{3}{*}{8.} & \multirow{3}{*}{$\begin{array}{l}\text { Number } \\
\text { of dorsal C }\end{array}$} & 0 & 20 & 40 \\
\hline & & 1 & 25 & 50 \\
\hline & & 2 & 5 & 10 \\
\hline \multirow{2}{*}{9.} & \multirow{2}{*}{$\begin{array}{l}\text { Number } \\
\text { of glottal C }\end{array}$} & 0 & 49 & 98 \\
\hline & & 1 & 1 & 2 \\
\hline \multirow{4}{*}{10.} & \multirow{4}{*}{ Initial C } & labial & 11 & 22 \\
\hline & & coronal & 31 & 62 \\
\hline & & dorsal & 7 & 14 \\
\hline & & glottal & 1 & 2 \\
\hline \multirow{4}{*}{11.} & \multirow{4}{*}{ Final C } & labial & 4 & 8 \\
\hline & & coronal & 22 & 44 \\
\hline & & dorsal & 17 & 34 \\
\hline & & labio-dorsal & 7 & 14 \\
\hline
\end{tabular}

the place dimension offers two preferred feature settings. Manner of articulation supplies material for formulating a wider range of phonotactic preferences. 


\subsubsection{Manner of articulation}

Categories used in the description of the manner features are based on Gimson (2014). Five manner-describing parameters are proposed, which lead to four preferences. In analogy to place of articulation, distances (12) are measured according to Dziubalska-Kołaczyk $(2009,2014)$. Here, a distance of one holds between two consecutive manners: plosives - affricates - fricatives nasals - liquids - glides. The values for CCCs are obtained by averaging over distances for two pairs of consonants. To take an example of /str/, C1$\mathrm{C} 2($ fricative-plosive $)=2, \mathrm{C} 2-\mathrm{C} 3($ plosive-liquid $)=4,(2+4) / 2=3$. This feature also uses broader manner categories, namely obstruents and sonorants, found in parameters (14-16). The parameter labelled number of obstruents records the proportion of obstruents to sonorants in a cluster, while initial $C$ and final $C$ specify the distribution of sonorants and obstruents clusterinitially and finally. In (14), percentages are calculated for CCs and CCCs separately due to varying cluster size. The presence of one obstruent in a double and triple cluster corresponds to one half and one third respectively. Parameters (14-16) provide partially overlapping information due to a binary distinction between sonorants and obstruents. For instance, if a double cluster contains only one obstruent segment (14) found in C1 (15), then C2 must obviously be represented by a sonorant; a phonotactic constraint repeated in (16). In this sense, these parameters provide a better insight into the structure of CCCs. Nevertheless, in order to use a set of parameters comparable to the place feature, (14-16) are all considered to be relevant.

The last measure proposed for the manner feature is articulatory opening. (13) corresponds to the sonority hierarchy (e.g. Goldsmith 1990) by specifying the degree of opening of the vocal tract from the first to the last segment. Possible patterns involve: increase, in which articulatory opening increases from $\mathrm{C} 1$ to $\mathrm{C} 2 / \mathrm{C} 3$ (e.g. plosive + glide $/ \mathrm{kw} /$, fricative + nasal $/ \mathrm{sm} /$, nasal + glide $/ \mathrm{nj} /$ ), decrease, in which a larger constriction is found towards the last segment (e.g. fricative + plosive /sk sp st/), and plateau characterised by an identical degree of stricture (i.e. fricative + fricative /sf sv/). In order to account for the structure of CCCs, a plateau-like pattern is established. All three-member clusters involve a combination of two gestures; decrease + increase (i.e. fricative + plosive + liquid /skj spl str/), which can be seen as a form of plateau but different from true plateaus found in CCs. Therefore, the pattern is kept distinct from the remaining articulatory types. 
Table 5. The manner of articulation feature.

\begin{tabular}{|c|c|c|c|c|}
\hline & Parameters & Patterns & No & $\%$ \\
\hline \multirow{6}{*}{12.} & \multirow{6}{*}{ Distance } & 0 & 2 & 4 \\
\hline & & 1 & 3 & 6 \\
\hline & & 2 & 10 & 20 \\
\hline & & 3 & 12 & 24 \\
\hline & & 4 & 14 & 28 \\
\hline & & 5 & 9 & 18 \\
\hline \multirow{4}{*}{13.} & \multirow{4}{*}{$\begin{array}{l}\text { Articulatory } \\
\text { opening }\end{array}$} & increase & 36 & 72 \\
\hline & & decrease & 3 & 6 \\
\hline & & plateau & 2 & 4 \\
\hline & & plateau-like & 9 & 18 \\
\hline \multirow{4}{*}{14.} & \multirow{4}{*}{$\begin{array}{l}\text { Number } \\
\text { of obstruents }\end{array}$} & $\mathrm{CC}: 2 / 2$ & 5 & 12 \\
\hline & & $1 / 2$ & 33 & 80 \\
\hline & & $0 / 2$ & 3 & 7 \\
\hline & & CCC: $2 / 3$ & 9 & 100 \\
\hline \multirow{2}{*}{15.} & \multirow{2}{*}{ Initial C } & obstruent & 47 & 94 \\
\hline & & sonorant & 3 & 6 \\
\hline \multirow{2}{*}{16.} & \multirow{2}{*}{ Final C } & obstruent & 5 & 10 \\
\hline & & sonorant & 45 & 90 \\
\hline
\end{tabular}

As shown in Table 5, several criteria in English initial phonotactics conspire to form clusters adhering to the SSG. The role of sonority is demonstrated on the basis of parameters (13-16), for which (strong) preferences are formulated. The majority of clusters exhibit an increase in the vocal tract opening from the first to the last segment (13). This preference is also reflected in the tendency for clusters to contain at least one obstruent (14), especially in C1 (15), and to end with a sonorant (16). The results obtained for articulatory distances are inconclusive; medial and larger distances seem to be favoured (distance $=2,3,4)$, but no clear preference can be stated.

\subsubsection{Voice}

The final set of parameters captures the voicing profile of segments forming a cluster. Parameters initial $C$ (17) and final $C$ (18) specify the voice feature 
for the first $(\mathrm{C} 1)$ and the last $(\mathrm{C} 2 / \mathrm{C} 3)$ consonant, while voice agreement captures a voicing profile throughout a cluster. For (19), the following patterns are distinguished; total and none, in which all adjacent segments either agree in voicing (e.g. voiceless + voiceless $/ \mathrm{sk} /$, voiced + voiced $/ \mathrm{bl} /$ ) or disagree in voicing (i.e. voiceless + voiced /fj pr sw/) respectively. All CCCs represent a pattern of partial agreement as they involve both total agreement in $\mathrm{C} 1 \mathrm{C} 2$ (voiceless + voiceless /sp st sk/) and no agreement in $\mathrm{C} 2 \mathrm{C} 3$ (voiceless + voiced $/ \mathrm{pj}$ tr kl/). The results obtained for the voice feature are summarized below.

Table 6. The voice feature.

\begin{tabular}{lllrr}
\hline & Parameters & Patterns & No & $\%$ \\
\hline 17. & Initial C & +voice & 15 & 30 \\
& & -voice & 35 & 70 \\
\hline 18. & Final C & +voice & 46 & 92 \\
& & -voice & 4 & 8 \\
\hline 19. & Voice agreement & total & 19 & 38 \\
& & partial & 9 & 18 \\
& & none & 22 & 44 \\
\hline
\end{tabular}

The distribution of [+voice] and [-voice] segments in clusters shows that they generally preserve the preferred sonority profile. Voiced segments are more sonorous than their voiceless counterparts; a generalization captured by, e.g. Jespersen (1904), Vennemann (1988), Parker (2002). Among clusters under investigation, most start with voiceless segments, and end with a voiced one. On this basis, the preferred voice setting for initial phonotactics in English can be stated: [-voice] cluster-initially (17), and [+voice] clusterfinally (18). This observation is reflected in (19), where voice disagreement between adjacent consonants prevails.

\section{Deriving weights}

On the basis of the obtained percentages (e.g. 92\% clusters have [+voice] specification cluster-finally, 18), the empirically established scores (e.g. score $=0.92)$ and their sum $(\Sigma)$ are provided. Table 7 presents a tentative ranking of the established preferences based on the number of clusters adhering to a given pattern. 
Table 7. Ranking of preferences: the first approach.

\begin{tabular}{cllc}
\hline Rank & Features & Preferences & Clusters \% \\
\hline \multirow{2}{*}{1} & \multirow{2}{*}{ complexity } & $\begin{array}{l}(2) \\
(3)\end{array}$ & full compositionality \\
& & total identity avoidance & 100 \\
\hline 2 & place & $(9)$ no glottal C & 98 \\
\hline 3 & manner & $(15)$ obstruent C cluster-initially & 94 \\
\hline 4 & voice & $(18)$ voiced C cluster-finally & 92 \\
\hline 5 & manner & $(16)$ sonorant C cluster-finally & 90 \\
\hline 6 & complexity & $(1)$ CC size & 82 \\
\hline 7 & manner & $(14)$ 1 obstruent C in CC & 80 \\
\hline 8 & manner & $(13)$ increase in articulatory opening & 72 \\
\hline 9 & voice & $(17)$ voiceless C cluster-initially & 70 \\
\hline 10 & place & $(10)$ coronal C cluster-initially & 62 \\
\hline
\end{tabular}

Let us now approach the ranking from a slightly different perspective by comparing the distribution of the 11 preferences among the highest- and lowest-scoring clusters. Both groups represent - what we would informally refer to as - "best" and "worst" clusters in terms of the degree to which they follow the established parameters, in particular preferences (1-3), (9), (10), (13-18). To compare clusters found at each extreme end of the scale, randomly we decided on a subset of clusters constituting $10 \%$ of the dataset, resulting in five "best" and "worst" CCs; the highest-scoring $/ \theta \mathrm{j}>\mathrm{sj}>\mathrm{tj}>\mathrm{pl}>$ $\theta \mathrm{w} /$, and lowest-scoring /sk $>\mathrm{mj}>\mathrm{sp}>\mathrm{sf}>\mathrm{st} /$ (where " $>$ " means "better than'). For these two classes, individual percentage scores and their sum are presented in Table 8. The sum $\Sigma$ reflects the cluster's degree of adherence to the 19 parameters, all of which have the same weight, and contribute equally to the total value.

The most extreme total scores amount to 12.82 (for $/ \theta \mathrm{j} /$ ) and 8.74 (for /st/), where 19 constitutes the maximum. Mean percentage scores $(=12.82 /$ $19=67.4,8.74 / 19=46$ ) show that word-initial clusters in English spread over the range between $67 \%$ and $46 \%$ (mean $=57 \%$ ), which suggests that there is no perfect cluster which would score highest on all parameters. This logical limitation results from the fact that some parameters are mutually exclusive. For instance, preferences for $[-$ voice $]$ in $\mathrm{C} 1$ and [+voice] clusterfinally preclude the preference for voice agreement. Similarly, there is no 
cluster which would be dispreferred on all parameters. Even the lowestscoring /st/ follows some preferences in being, among others, a CC sequence starting with a voiceless obstruent.

The highest-scoring clusters are characterised by an increase in articulatory opening (13), an obstruent+sonorant structure (14-16), as well as a voicing profile in which voiceless and voiced segments tend to be found clusterinitially and finally respectively (17-18). Moreover, $/ \theta \mathrm{j}$ sj tj pl $\theta \mathrm{w} /$ start with a coronal segment (10), and follow a set of (almost) absolute preferences in that they are doubles (1) composed of existing singletons (2), devoid of two identical and glottal segments $(3,9)$. The other extreme of the list is well represented by $\mathrm{sC}$ clusters, which generally pose problems for any phonological theory.

A close inspection of the scores assigned to the 'worst' clusters reveals that they follow some preferences, although fewer compared to the previous group. /st sf sp mj sk/ adhere to preferences (1-3) and (9), common for all or the vast majority of English initial clusters, and generally start with a voiceless coronal obstruent, in such a way satisfying (17), (10) and (15), respectively. These clusters violate only a subset of the preferences, namely increase in articulatory opening (13), presence of one obstruent $C$ (14), presence of a sonorant $C$ cluster-finally (16), and presence of a voiced $C$ clusterfinally (18). It is thus assumed that the clusters' low rank can be attributed to the fact that /st sf sp mj sk/ fail to observe (13), (14), (16), and (18). At the same time, /st sf sp mj sk/ follow another set of preferences that guarantee the group's preservation in the lexicon. The suggested interpretation is that the preferences proposed in Section 3.3 must vary in weight.

Here, I advocate the idea that the manner and voicing features leading to a sonority-based profile play a greater role in the formation of word-initial clusters in English than, for instance, the place feature (a question to be tested statistically in Section 4.1). The same conclusion can be drawn when analysing CCCs. Longer clusters have higher scores than the $\mathrm{sC}$ group. This, again, points to the fact that in spite of a strong preference for CC $(82 \%)$, features pertaining to sonority constitute a more critical criterion in cluster formation in English. Cluster size, which expresses the universal preference for $\mathrm{CV}$, is seen as a weaker building block of clusters than sonority. What makes CCCs different from lowest-scoring $\mathrm{sC}$ clusters is that the first end in a voiced segment, in particular a sonorant. What makes /skj skl skr skw spj spl spr stj str/ different from the highest-scoring class is that the first involve a plateau-like articulation. These differences support the hypothesis that pa- 


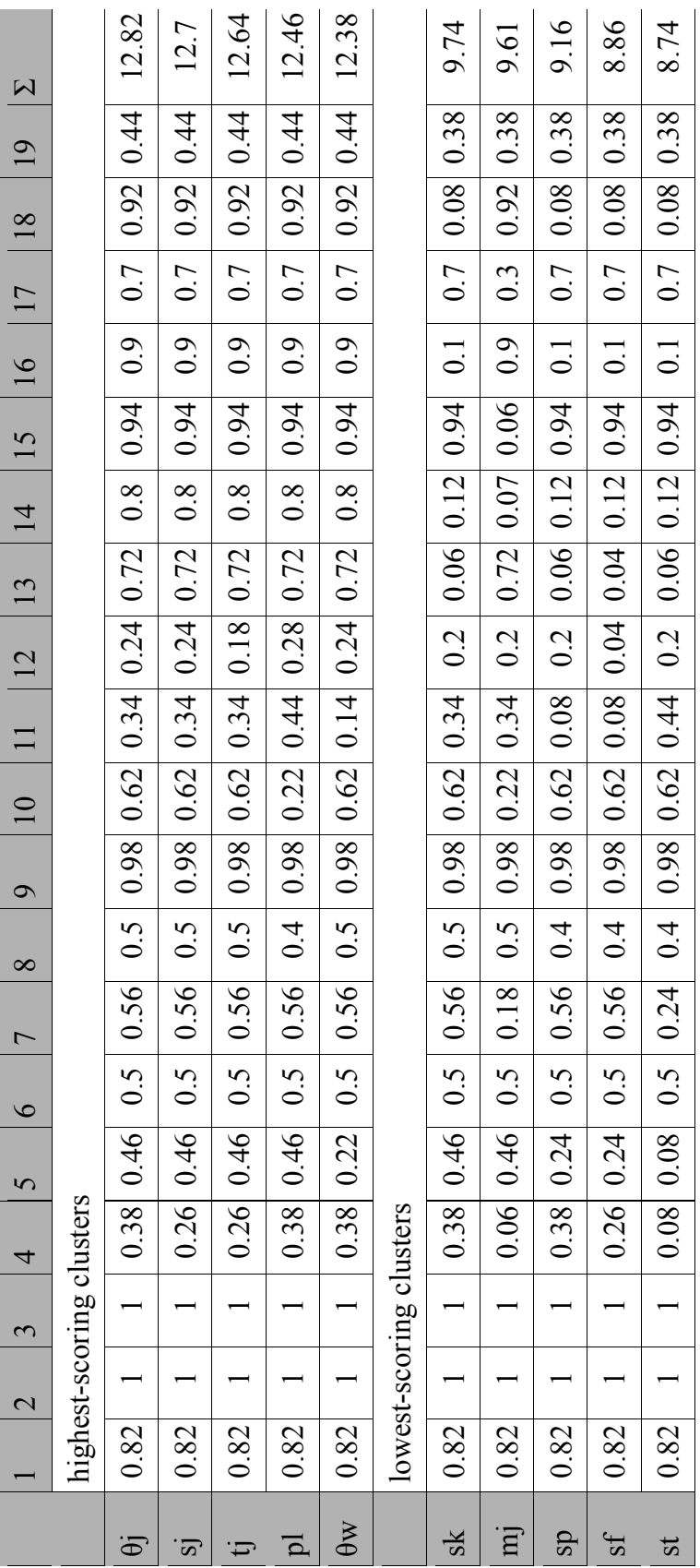


rameters (13), (16) and (18) expressing the manner and voicing properties have greater weight in cluster formation. To follow this logic, the original ranking is rearranged to account for the critical preferences that show to be significant in the discrimination of 'best' and 'worst' clusters (ranks 1-4).

Table 9. Ranking of preferences: the second approach.

\begin{tabular}{cllc}
\hline Rank & Features & Preferences & Clusters \% \\
\hline 1 & voice & $(18)$ voiced C cluster-finally & 92 \\
\hline 2 & manner & $(16)$ sonorant C cluster-finally & 90 \\
\hline 3 & manner & $(14)$ 1 obstruent C in CC & 80 \\
\hline 4 & manner & $(13)$ increase in articulatory opening & 72 \\
\hline 5 & complexity & $(2)$ full compositionality & 100 \\
& & $(3)$ total identity avoidance & 100 \\
\hline 6 & place & $(9)$ no glottal C & 98 \\
\hline 7 & manner & $(15)$ obstruent C cluster-initially & 94 \\
\hline 8 & complexity & $(1)$ CC size & 82 \\
\hline 9 & voice & $(17)$ voiceless C cluster-initially & 70 \\
\hline 10 & place & $(10)$ coronal C cluster-initially & 62 \\
\hline
\end{tabular}

The modified ranking shifts the features of voice and manner to the very top. The subsequent section of the paper aims at testing the proposal by using statistical methods.

\subsection{Statistical weights}

In order to determine the role of the respective parameters in cluster formation, the Principal Component Analysis (PCA) was performed. This method is considered to be most adequate for the purpose of the present study as it allows us to reveal the structure of the data with a large number of variables, i.e. 19 parameters. All variables are correlated and almost all are significantly correlated, with the exception of parameter (8), the number of dorsal $C$, which is not correlated with any other parameter. The resulting principal components (Comp 1-19) are linearly uncorrelated; the first com- 
ponent has the largest variance, while each succeeding component accounts for the data which is not explained by the preceding component. As shown in the scree plot in Figure 1, there is no single component which would explain most of the variability in the data. Comp 1 explains $33 \%$ (proportion of variance $=0.335801$ ) of the variability, Comp 2, seen as supplementary to Comp 1 , explains $26 \%$ (proportion of variance $=0.259216$ ) of the variability that is not covered by the first component. Comp 3 explains merely 5\% (proportion of variance $=0.05$ ) of the data which is not accounted for by Comp 2. The number of components corresponds to the number of variables used. However, here we depict only the first 10 components due to very low variance level for Comp 5-12.

For statistical interpretation, a small number of components explaining large amounts of the overall variance should be determined. Here, the visual assessment of the scree plot suggests that the first two components are dominant; they stand out amongst other components and jointly capture $60 \%$ of the variability of the data (cumulative proportion $=0.5950170$ ). The remaining factors (Comp 3-10) explain a smaller proportion of variability and, therefore, do not enter a further analysis.

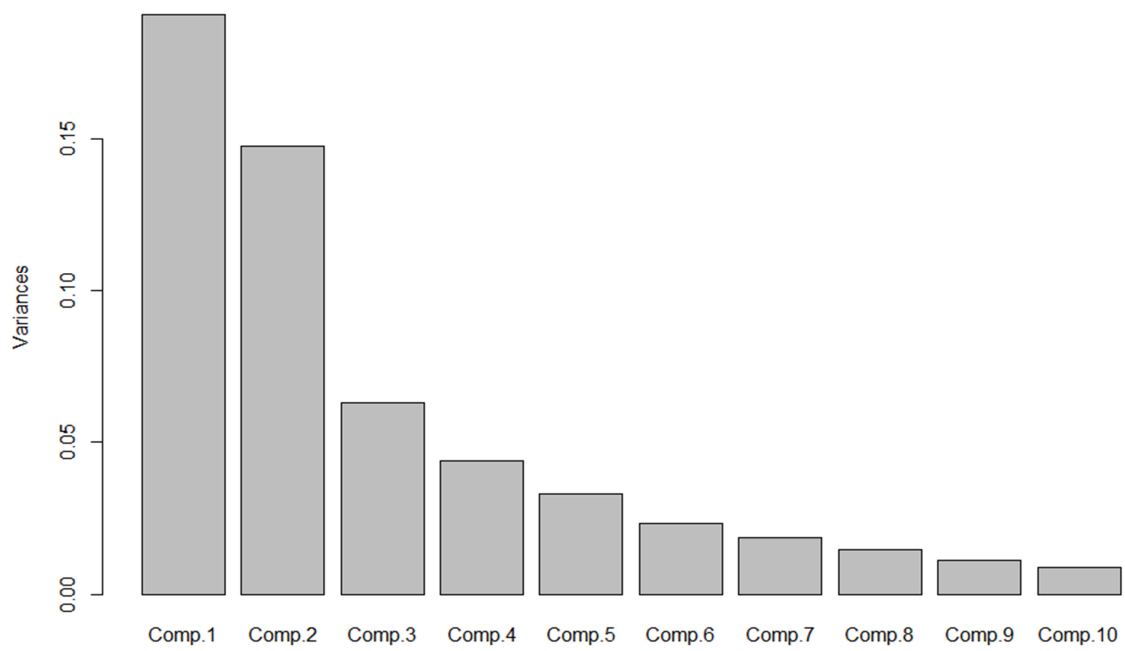

Figure 1. Variance of principal components. 
Comp 1 and 2 are thus used to weigh parameters (1-19) on the basis of the PCA loadings. The loadings obtained for Comp 1 are interpreted as more prominent, followed by Comp 2 loadings. The summary of the results is provided in Table 10.

Table 10. PCA loadings for the first two principal components.

\begin{tabular}{llllllll}
\hline \multicolumn{1}{l}{ Comp 1 } & \multicolumn{7}{c}{ Comp 2 } \\
\hline 1 & 0.011 & 11 & -0.104 & 1 & $-0.583(33 \%)$ & 11 & 0.026 \\
2 & & 12 & -0.062 & 2 & 12 & 0.049 \\
3 & & 13 & $-0.427(18 \%)$ & 3 & & 13 & $-0.461(21 \%)$ \\
4 & 0.024 & 14 & $-0.495(24 \%)$ & 4 & 0.006 & 14 & $0.429(18 \%)$ \\
5 & -0.056 & 15 & -0.057 & 5 & -0.110 & 15 & 0.260 \\
6 & & 16 & $-0.523(27 \%)$ & 6 & & 16 & 0.037 \\
7 & 0.078 & 17 & 0.098 & 7 & -0.108 & 17 & 0.255 \\
8 & 0.006 & 18 & $-0.464(21 \%)$ & 8 & -0.068 & 18 & 0.032 \\
9 & 0.031 & 19 & -0.014 & 9 & 0.022 & 19 & -0.196 \\
10 & $0.211(4 \%)$ & & & 10 & 0.242 & & \\
\hline
\end{tabular}

Absolute values of the loadings (ranging from 0 to 1 ) reflect the role that an individual parameter plays in the discrimination of clusters. That is, an increase in the value corresponds to an increase in weight. Since the values obtained are relatively low (the highest value $=0.583$ ) and there is no single preference which would stand out in terms of its PCA loading, several preferences with the highest values (shaded cell marking) are assumed for data interpretation (cut-off point $>0.4$ ). Percentage values show the degree to which a parameter contributes to the sum, i.e. serves as a relevant factor in cluster discrimination. The largest loadings are found for (13), (14), (16), (18) in Comp 1 and (1), (13), (14) in Comp 2. On this basis, the final ranking of the parameters can be proposed: namely $16>14>18>13$ for Comp 1 and $1>13>14$ for Comp 2. The results are summarized in Table 11 . 
Table 11. Statically-based feature weights for components 1 and 2: the final proposal.

\begin{tabular}{|c|c|c|c|}
\hline Rank & Feature & & Preference \\
\hline \multicolumn{4}{|c|}{ Comp 1} \\
\hline 1 & manner & $(16)$ & sonorant $\mathrm{C}$ cluster-finally \\
\hline 2 & manner & (14) & 1 obstruent $\mathrm{C}$ in $\mathrm{CC}$ \\
\hline 3 & voice & $(18)$ & voiced $\mathrm{C}$ cluster-finally \\
\hline 4 & manner & (13) & increase in articulatory opening \\
\hline \multicolumn{4}{|c|}{ Comp 2} \\
\hline 1 & complexity & $(1)$ & CC size \\
\hline 2 & manner & (13) & increase in articulatory opening \\
\hline 3 & manner & (14) & 1 obstruent $\mathrm{C}$ in $\mathrm{CC}$ \\
\hline
\end{tabular}

Decompositionality (2), identity avoidance (3) and presence of a labial C (6) are demonstrated to be factors not contributing to cluster discrimination. Interestingly, the ranking for Comp 1 corresponds with the tentative ranking proposed earlier in Table 9, where preferences (18), (16), (14) and (13) were found at the top of the hierarchy. Statistically, I confirm that the manner and voice features have greater weight than the place features. Parameters leading to a sonority-obeying profile are preferred in English word-initial phonotactics as they contribute to cluster discrimination. It must be noted that the place parameter defined as a preference (i.e. coronality cluster-initially, 10) is shown to come next in terms of rank in Comp 1 (PCA loading $=0.211$ ). Based on its loading, (10) is ranked after (13) for Comp 1 but it accounts for only $4 \%$ of the variability in the data. For the time being, we leave the issue aside, and propose a statistical ranking based on the $>0.4$ threshold.

The Cluster Analysis (CA) constitutes another statistical way of illustrating the inherent structure of phonological clusters. In the section to follow, two types of clusters are distinguished to avoid confusion. The term "statistical cluster" (SC) is used to describe the groups of clusters generated by the model, while "phonological clusters" (PC) refer to the target wordinitial clusters in the dataset. Phonological clusters (here labelled as numbers for clarity of presentation) and corresponding parameters (1-19) were 


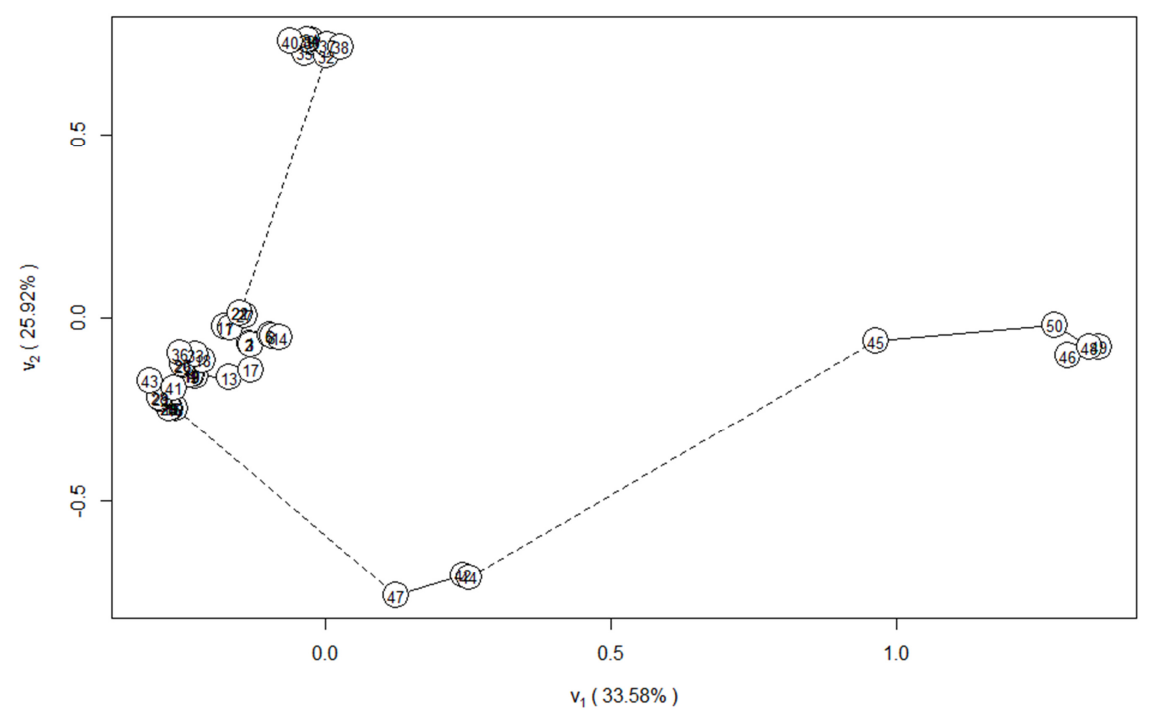

Figure 2. Statistical clustering of word-initial phonotactics in English.

entered into the R software (R Core Team 2015) as variables. Several types of different clustering methods were used: Ward's minimum variance method, the single linkage method (which is closely related to a minimal spanning tree) with different distance (dissimilarity) measures: Euclidean, and Manhattan. Finally, a minimal spanning tree (given in Figure 2) was generated; it shows (dis)similarity between the statistical clusters found. Crucially, all the methods distinguish four identical sets of statistical clusters. In Table 12, column SC stands for the joint (here identical) result obtained for all the methods. Each cluster is labelled as a number PC $=1-50$, and assigned to a particular statistical group $\mathrm{SC}=1-4$. The division into statistical clusters in Figure 2 is calculated on the basis of all variables; however, the chart is based only on the first two components (Comp 1 and 2 on the $\mathrm{x}$ and y axes respectively).

For the four statistical clusters, a set of characteristics can be provided. Dotted lines show the distance between various statistical clusters, while solid lines denote clusters within one group. The larger the distance, the greater the dissimilarity between the groups or individual clusters within each respective group. To start with the right-most edge of the plot, SC4 encompas- 


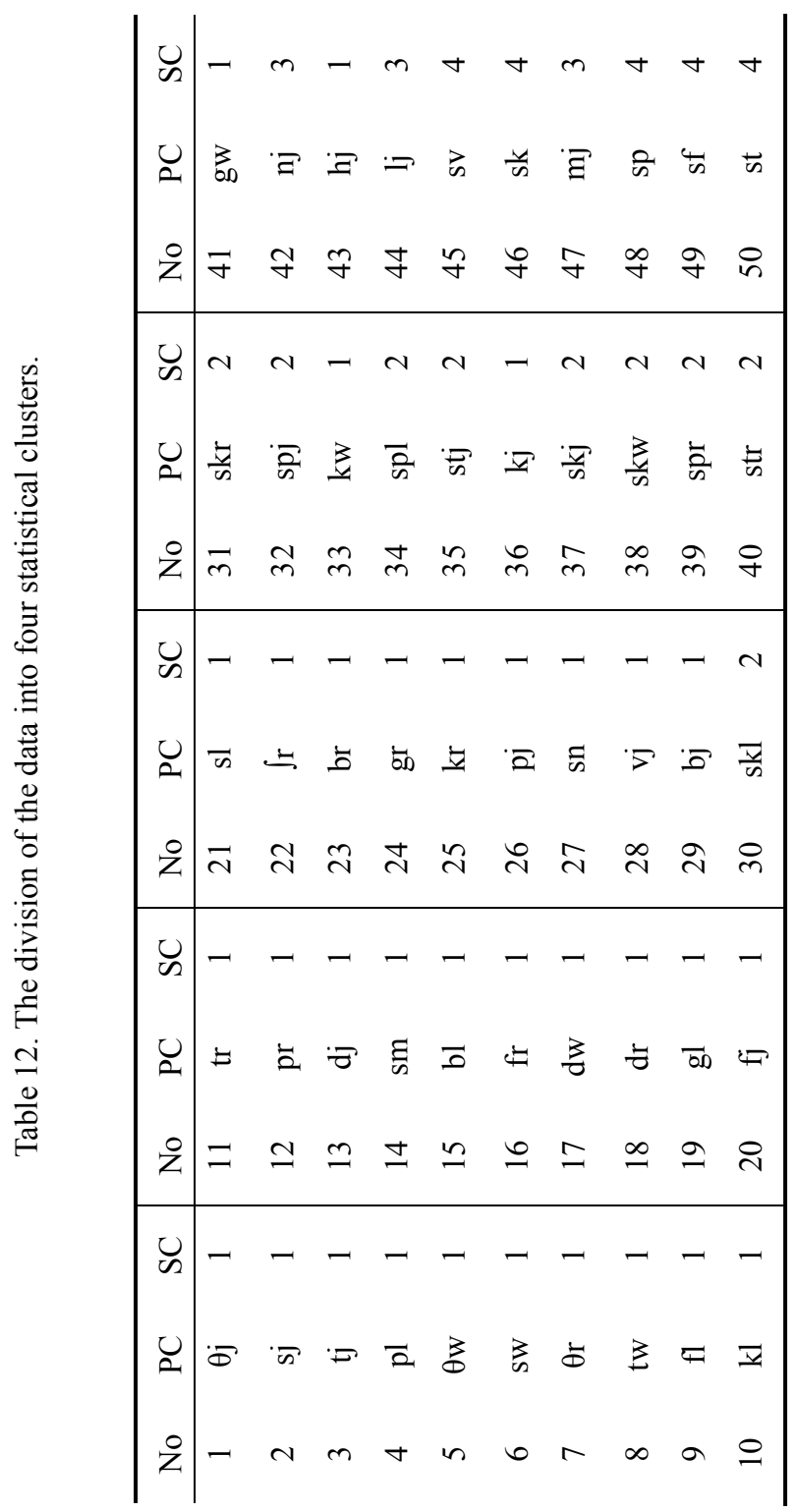


ses all $\mathrm{s}+\mathrm{C}$ clusters /sp st sk sf sv/; doubles mainly composed of two voiceless obstruents with a plateau or decreasing sonority profile. /sv/ (labelled 45) is part of the group but is most dissimilar from the remaining phonological clusters due to voicing cluster-finally. The closest to SC4 is the group of sonorant sequences /mj nj lj/ subsumed under SC3. Here, $/ \mathrm{mj} /$ (labelled 47) is shown as dissimilar from $/ \mathrm{nj} /$ and $/ \mathrm{lj} /$, which is due to the place feature in $\mathrm{C} 1$. Parameter (10) coronal C cluster-initially, which explains the emerging distance, was discussed earlier as a preference and as having a potential rank 5 in Comp 1. The relevance of (10) in phonotactics is supported by contributions to the special status of coronality (Paradis and Prunet 1991; Hall 1997).

The densest areas of the data space are represented by SC1 (left-most group) and SC2 (top-most group). SC2 contains all triple clusters composed of two voiceless obstruents and followed by a voiced sonorant, resulting in a plateau-like sonority profile. The defining properties of this class are thus similar to s+stop clusters in SC4, however, as shown in the plot, the two statistical clusters are the most distant ones. Obviously, the dissimilarity between SC4 and SC1 is due to cluster size (CCCs vs. CCs respectively), which supports the role of parameter (1) in English initial phonotactics. Finally, SC1 contains the remaining 29 phonological clusters.

Based on the intervals of statistical distribution, the "regular" SC1 group merges between $/ \mathrm{mj} \mathrm{nj} \mathrm{lj} /$ and CCCs. The distance between $\mathrm{SC} 1$ and sonorant clusters ( $\mathrm{SC} 3$ ) results from the manner and voice properties of the first consonant (voiceless obstruent vs voiced sonorant). The distance between SC1 and triple sequences ( $\mathrm{SC} 2)$ is attributed to cluster length ( $\mathrm{CC}$ vs $\mathrm{CCC}$ ). Interestingly, the most dissimilar statistical cluster contains sC sequences. SC4 is found furthest apart from other statistical clusters, in particular from $\mathrm{SC} 2$ containing triple sequences. This again suggests that the parameter of size (1) is a weaker predictor of cluster dissimilarity than parameters $(18,16)$ which define the final consonant in a cluster. The interpretation adopted here is that a lack of a voiced sonorant cluster-finally contributes mostly to phonological dissimilarity among all the collected clusters. /sp st sk sf sv/ largely violate preferences ranked 1 and 3, which guarantee a sonority-based transition from $\mathrm{C} 1$ to a vowel, and which are some of the basic defining properties of all the remaining statistical and phonological clusters. This observation casts a new light on the relation between linguistic universals and language-specific preferences. English word-initial phonotactics favours phonological clusters constructed on the basis of an increasing sonority profile, rather than on cluster size. CCCs, in spite of being a severe violation of the phonotactic universal 
favouring the basic syllable template $\mathrm{CV}$, display a sonority rise $(\mathrm{C} 2 \mathrm{C} 3$ : stop + approximant), and are therefore more similar to other clusters than the sonority-violating $\mathrm{sC}$ class.

\section{Conclusions}

The goal of this contribution was to test a new approach to phonotactics, an extension of an earlier model, on English data. The proposed method allowed us to trace idiosyncratic properties of consonant clustering, formulate novel positional constraints, and eventually answer questions related to factors motivating the structure of consonant clusters in a given language. The paper demonstrated that phonological features of place, manner and voice do not contribute identically to the structure of initial consonant clusters in English. A quantitative analysis of an exhaustive inventory of clusters in terms of the 19 parameters led to proposing 11 phonotactic preferences valid for the word-initial position. Weights assigned statistically to the preferences and rankings suggest that the features of manner of articulation and voicing, which conspire to create sonority-based clusters, play a greater role in English initial phonotactics than the place features do.

Future work on feature weighting will involve testing the results in experiments on perception and production, and juxtaposing the preferences with frequency data. Moreover, the extended approach is planned to be tested on other languages and different word positions with a view to discovering typologically-based preferences governing cluster formation.

\section{Acknowledgements}

I would like to thank Dafydd Gibbon, Małgorzata Haładewicz-Grzelak and Janina Mołczanow for valuable comments on earlier versions of this work, Waldemar Wołyński for help with statistical processing of the data, and Adam Olender for remarks on statistics. 


\section{References}

Clements, G.N. 1985. "The geometry of phonological features". Phonology Yearbook 2. 225-252.

Clements, G.N. 1990. "The role of the sonority cycle in core syllabifcation". In: Kingston, J. and M.E. Beckman (eds.), Papers in laboratory phonology I: Between the grammar and physics of speech. Cambridge: CUP. 283-333.

Dresher, B.E. 2009. The contrastive hierarchy in phonology. Cambridge: Cambridge University Press.

Dziubalska-Kołaczyk, K. 2009. "NP extension: B\&B phonotactics”. Poznań Studies in Contemporary Linguistics 45(1). 55-71.

Dziubalska-Kołaczyk, K. 2014. "Explaining phonotactics using NAD”. Language Sciences 46. 6-17.

Gimson, A.C. 2014. An introduction to the pronunciation of English. (8th ed.) London: Edward Arnold.

Goad, H. 2011. "The representation of sC clusters". In: van Oostendorp, M., C. Ewen, E. Hume and K. Rice (eds.), The Blackwell companion to phonology. Oxford: WileyBlackwell. 898-923.

Goad, H. 2012. "sC Clusters are (almost always) coda-initial". The Linguistic Review 29(3). 335-373.

Goldsmith J.A. 1990. Autosegmental and metrical phrenology. Oxford: Blackwell.

Greenberg, J.H. 1978. "Some generalizations concerning initial and final consonant clusters". In: Greenberg, J.H. (ed.), Universals of human language. Stanford: Stanford University Press. 243-279.

Hayes, B. 1989. "Compensatory lengthening in moraic phonology". Linguistic Inquiry 20. 253-306.

Hall, T.A. 1997. The phonology of coronals. Amsterdam: John Benjamins.

Harris, J. 1990. "Segmental complexity and phonological government". Phonology Yearbook 7. 255-300.

Hoole, P., L. Bombien, M. Pouplier, C. Mooshammer and B. Kühnert (eds.). 2012. Consonant clusters and structural complexity. Berlin: Mouton de Gruyter.

Hyman, L. 1977. "On the nature of linguistic stress". In: Hyman, L. (ed.), Studies in stress and accent. (Southern California Occasional Papers in Linguistics 4.) Los Angeles: USC Department of Linguistics. 37-82.

Jespersen, O. 1904. Lehrbuch der Phonetik. (2nd ed.) Leipzig, Berlin: Teubner.

Jones, D. 2011. Cambridge English pronouncing dictionary. (18th ed.) Cambridge: Cambridge University Press.

Ladefoged, P. 2011. A course in phonetics. Boston: Thomson Higher Education.

Leben, W. R. 1973. Suprasegmental phonology. (PhD dissertation, MIT.)

Kaye, J. 1992. "Do you believe in magic? The story of s+C sequences". SOAS Working Papers in Linguistics and Phonetics 2. 293-313.

Kaye, J., J. Lowenstamm and J-R. Vergnaud. 1990. "Constituent structure and government in phonology". Phonology 7. 193-231.

Maddieson, I. 2011. "Syllable structure". In: Dryer, M. and M. Haspelmath (eds.), The world atlas of language structures online. Munich: Max Plank Digital Library. $<$ http://wals.info/>. 
McCarthy, J.J. 1986. “OCP effects: gemination and antigemmination”. Linguistic Inquiry 17. 207-263.

Morelli, F. 1999. The phonotactics and phonology of obstruent clusters in Optimality Theory. (PhD dissertation, University of Maryland, College Park, MD.)

Orzechowska, P. and R. Wiese. 2011. "Reconstructing the sonority hierarchy". Proceedings of ICPhS 2011, Hong Kong.

Orzechowska, P. and R. Wiese. 2015. "Preferences and variation in word-initial phonotactics: A multi-dimensional evaluation of German and Polish". Folia Linguistica 49(2). 439-486.

Paradis, C. and J-F. Prunet (eds.). 1991. The special status of coronals: Internal and external evidence. San Diego: Academic Press.

Parker, S. 2002. Quantifying the sonority hierarchy. (PhD dissertation, University of Massachusetts, Amherst, MA.)

R Core Team. 2015. The R project for Statistical Computing. $<$ http://www.R-project.org/>.

Roach, P. 2004. "British English: Received Pronunciation”. Journal of the International Phonetic Association 34(2). 239-245.

Roach, P. 2006. English phonetics and phonology: A practical course. Cambridge: Cambridge University Press.

Rochoń, M. 2000. Optimality in complexity: The case of Polish consonant clusters. Berlin: Akademie-Verlag.

Selkirk, E.O. 1984. "On the major class features and syllable theory". In: Aronof, M. and R.T. Oehrle (eds.), Language sound structure. Cambridge, MA: The MIT Press. 107-136.

Steriade, D. 1982 Greek prosodies and the nature of syllabification. (PhD dissertation, MIT.)

Trnka, B. 1966. A phonological analysis of present-day standard English. Alabama: University of Alabama Press.

Vennemann, T. 1988. Preference laws for syllable structure and the explanation of sound change. Berlin/Boston: Mouton de Gruyter.

Wells, J.C. 2009. Longman pronunciation dictionary. (3rd ed.) London: Pearson.

Wiese, R. 1988. Silbische und Lexikalische Phonologie. Studien zum Chinesischen und Deutschen. Tübingen: Max Niemeyer Verlag.

Zec, D. 1995. "Sonority constraints on syllable structure". Phonology 12. 85-129.

\author{
Address for correspondence: \\ Paula Orzechowska \\ Faculty of English \\ Adam Mickiewicz Unviersity \\ Collegium Novum \\ al. Niepodległości 4 \\ 61-874 Poznań \\ Poland \\ paulao@wa.amu.edu.pl
}

